\title{
Preliminary study to investigate the Delboeuf illusion in ring-tailed lemurs (Lemur catta): Methodological challenges
}

\author{
Maria Santacà ${ }^{*}$, Barbara Regaiolli², Maria Elena Miletto Petrazzini', \\ Caterina Spiezio ${ }^{2}$ and Christian Agrillo ${ }^{1}$
}

\author{
${ }^{1}$ Department of General Psychology, University of Padova (Italy) \\ ${ }^{2}$ Research and Conservation Department, Parco Natura Viva - Garda Zoological Park (Bussolengo, Verona, Italy) \\ *Corresponding author (Email: santacamaria@gmail.com)
}

Citation - Santacà, M., Regaiolli, B., Miletto Petrazzini, M. E., Spiezio, C. \& Agrillo, C. (2017). Preliminary study to investigate the Delboeuf illusion in ring-tailed lemurs (Lemur catta): Methodological challenges. Animal Behavior and Cognition, 4(3), 365-377. doi: 10.26451/abc.04.03.13.2017

\begin{abstract}
Visual illusions are commonly used in animal cognition studies to compare visual perception among vertebrates. To date, researchers have focused their attention mainly on birds and mammals, especially apes and monkeys, but no study has investigated sensitivity to visual illusions in prosimians. Here we investigated whether lemurs (Lemur catta) perceive the Delboeuf illusion, a well-known illusion that occurs when subjects misperceive the relative size of an item because of its surrounding context. In particular, we adopted the spontaneous preference paradigm used in chimpanzees and observed lemurs' ability to select the larger amount of food. In control trials, we presented two different amounts of food on two identical plates. In test trials, we presented equal food portion sizes on two plates differing in size: If lemurs were sensitive to the illusion, they were expected to select the food portion presented on the smaller plate. In control trials, they exhibited poor performance compared to other mammals previously observed, being able to discriminate between the two quantities only in the presence of a 0.47 ratio. This result prevented us from drawing any conclusion regarding the subjects' susceptibility to the Delboeuf illusion. In test trials with the illusory pattern, however, the subjects' choices did not differ from chance. Our data suggest that the present paradigm is not optimal for testing the perception of the Delboeuf illusion in lemurs and highlight the importance of using different methodological approaches to assess the perceptual mechanisms underlying size discrimination among vertebrates.
\end{abstract}

Keywords - Visual illusions, Lemur catta, Size illusion, Comparative perception

The study of visual illusions in non-human animals has become a useful tool to compare visual perception in human and non-human animals. The assumption underlying these studies is that if two species share similar perceptual mechanisms, they are likely to exhibit a similar sensitivity to illusory patterns. Mainly non-human primates have been studied in this field. For instance, chimpanzees and rhesus monkeys are sensitive to the Ponzo illusion (Bayne \& Davis, 1983; Fujita, 1997); rhesus monkeys exhibit a human-like perception of the Zöllner illusion (Agrillo, Parrish, \& Beran, 2014a) and capuchin monkeys perceive the Müller-Lyer illusion (Suganuma, Pessoa, Monge-Fuentes, Castro, \& Tavares, 2007) and are partially sensitive to the Solitaire illusion (Parrish, Agrillo, Perdue, \& Beran, 2016). Illusory patterns have also been studied in non-primate species: Gray parrots can perceive the MüllerLyer illusion (Pepperberg, Vicinay, \& Cavanagh, 2008), chicks can perceive the Ebbinghaus illusion (Rosa Salva, Rugani, Cavazzana, Regolin, \& Vallortigara, 2013) and bamboo sharks can see illusory contours in the Kanizsa triangle (Fuss, Bleckmann, \& Schluessel, 2014). 
These studies suggest that vertebrates may share similar perceptual mechanisms. However, no firm conclusions can be made regarding the universality of perceptual mechanisms, as several species have yet to be studied. In addition, in some cases, no perception of illusory patterns was found (e.g., Solitaire illusion in chimpanzees and rhesus monkeys, Agrillo, Parrish, \& Beran, 2014b; Müller-Lyer illusion in bamboo sharks, Fuss et al., 2014), and a perception of reversed illusions was also reported, meaning that subjects were shown to perceive some sort of visual illusion but in the opposite direction to that typically perceived by human observers [e.g., Ebbinghaus illusion and Zöllner illusion in pigeons (Nakamura, Watanabe, \& Fujita, 2008; Watanabe, Nakamura, \& Fujita, 2011); Zöllner illusion in bantams (Watanabe, Nakamura, \& Fujita, 2013)].

One of the most commonly tested size illusions is called the "Delboeuf illusion." This occurs when subjects misperceive the relative size of an item because of its surrounding context. Figure 1 shows a classic version of the illusion: two identical test circles encompassed by a larger and smaller ring. In this case, most human participants estimate the test circle encompassed by the smaller ring as larger. Interestingly, this illusion seems to affect our food choice in natural contexts. It was found that human participants overestimate food portion sizes and hence underserve on smaller dishes (e.g., Davis, Payne, \& Bui, 2016; Van Ittersum \& Wansink, 2007; Wansink \& Cheney, 2005), showing that our perception of food size is influenced by the context in a Delboeuf-like fashion.
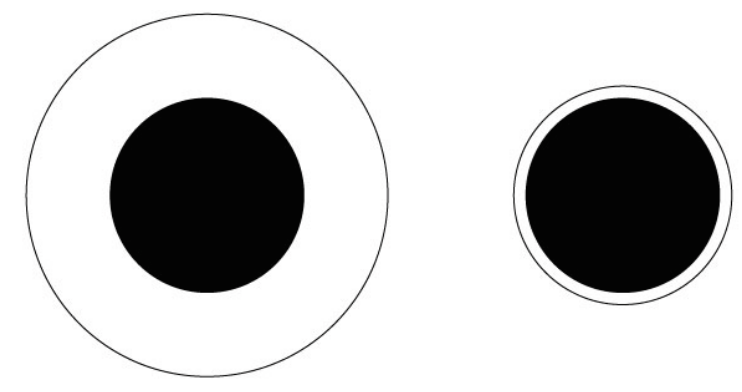

Figure 1. The Delboeuf illusion. This illusion occurs when two same-sized objects are perceived to be different depending upon the surrounding context. In this example, the black circles are physically identical, but human observers typically underestimate the size of the black circle encompassed by the larger ring and tend to overestimate the size of the black circle encompassed by the smaller ring.

The same perceptual bias affects chimpanzees' food choices. Parrish and Beran (2014) used the natural tendency of chimpanzees to reach for the larger food portion to assess the perception of the Delboeuf illusion in three adult subjects. Two different-sized food portions were presented on two identical plates in control trials in which subjects were expected to select the larger portion. In the test phase, chimpanzees were presented with two identical food portions on different-sized plates. If subjects perceived the illusion, they were expected to select the portion on the smaller plate. Results showed that chimpanzees significantly more often selected the food portion on the smaller plate. A further set of control trials showed that when chimpanzees were presented with a larger and a smaller food portion on a larger and smaller plate, respectively, they were able to select the larger portion of food, showing that they did not simply prefer the smaller plate when food sizes were not equivalent. A subsequent study using a training procedure also showed the perception of the Delboeuf illusion in capuchin monkeys (Parrish, Brosnan, \& Beran, 2015). Subjects were initially trained to distinguish between a larger and a smaller black circular stimulus. In the test phase, the same stimulus was encircled by a larger and a smaller ring. Subjects selected the stimulus encircled by the smaller ring at a rate greater than chance. On the contrary, data from rhesus monkeys tested in the same conditions were less clear, with some subjects showing a human-like perception of the Delboeuf pattern and others exhibiting a reverse illusion (Parrish et al., 2015). 
Susceptibility to this illusion has not been found in non-primate species. A recent study used the same methodological approach (spontaneous choice test with food portions as stimuli) adopted by Parrish and Beran (2014) to investigate dogs' sensitivity to the Delboeuf illusion. Control trials showed that dogs tried to maximize food intake, but no preference for either plate was shown in test trials with the illusory pattern, suggesting that dogs do not perceive the Delboeuf illusion (Miletto Petrazzini, Bisazza, \& Agrillo, 2017). This conclusion was supported by another study in which dogs underwent an extensive training requiring size discrimination: Subjects proved able to discriminate between two circles differing in size in control trials, but they did not select one array more than chance in test trials with the illusory pattern, reinforcing the idea that the perceptual biases affecting size judgments might differ between humans, apes, monkeys and other mammals (Byosiere et al., 2017).

As far as we are aware, no study has investigated visual illusions in prosimians. Investigating the Delboeuf illusion in prosimians would be particularly useful to assess similarities and differences in the perceptual bias underlying size perception between prosimians and other mammals. Lemurs in particular are among the most endangered species. Exploitation is pushing the ring-tailed lemur toward extinction in its natural environment, and a large number of lemurs are also kept illegally as pets (Reuter, LaFleur \& Clarke, 2017). Increasing our knowledge on lemurs' perceptual skills may help us develop proper enrichment programs for lemurs confined in natural parks.

In the present study, ring-tailed lemurs were observed in two experiments in their spontaneous preference to reach one of two plates containing food. In the control trials of Experiment 1, the two plates were identical in size, and physically different portions of food were presented ( 0.66 ratio between the smaller and the larger food portion). In the test phase, the same portion of food was shown on two plates differing in size. If subjects perceived the Delboeuf illusion, they were expected to select the portion on the smaller plate. Given that subjects did not show a significant preference for either plate in both control and test trials, in Experiment 2, we enlarged the physical difference between food portions ( 0.47 ratio) to ensure that subjects were actually trying to maximize food intake in the present experimental context.

\section{Method}

\section{Experiment 1}

Subjects. Nine adult ring-tailed lemurs (Lemur catta), five females and four males, participated in this study (Table 1). The lemurs belonged to a colony housed at Parco Natura Viva - Garda Zoological Park, Bussolengo (VR, Italy). The colony was housed in a $1.172 \mathrm{~m}^{2}$ naturalistic enclosure composed of a heated indoor area of $80 \mathrm{~m}^{2}$ and an outdoor area of $1.083 \mathrm{~m}^{2}$, connected to each other by two guillotine doors. The test room $\left(9 \mathrm{~m}^{2}\right)$ was connected to both areas of the enclosure with guillotine doors. Lemurs were fed twice daily with fruit and vegetables, and water was available ad libitum. Subjects were never food or water deprived prior to or during testing. Subjects were widely familiar with the test room and habitually entered individually and retrieved food in isolation and without visual contact with other conspecifics, in order to avoid competition and social learning. None of the subjects had previous experience with size discrimination tasks.

Stimuli and apparatus. The food used consisted of brown vegetable jellies ("primate jelly"), a highly preferred food for the lemurs of the colony. The jellies were cut with an egg-slicer to equalize their thickness and shaped with glue tops ("Coccoina") to get a round form. Then, each jelly was placed in the middle of a plate.

Laminated white plastic papers were used as plates and presented in a tray $(26 \mathrm{~cm}$ width $\mathrm{x} 22 \mathrm{~cm}$ length) made by black plastic material (poliplack), in order to maximize the contrast between the plates and the surrounding context. Two different sizes of plates were used: larger plates $(8 \mathrm{~cm}$ in diameter) and smaller plates $(3.66 \mathrm{~cm}$ in diameter). The tray was fixed to a wooden parallelepiped (10 width $\mathrm{x} 26$ length $\mathrm{x} 3$ height $\mathrm{cm}$ ) so that the lemurs could not move it when selecting one option. Such support was slightly inclined in order to improve the visibility of the stimuli from subjects' points of view. In particular, the tray was inclined by an 8.37 degree angle in the direction of the subjects. 
Table 1

Information of the Subjects Participating in the Study and Individual Performance for Experiment 1 and Experiment 2

\begin{tabular}{|c|c|c|c|c|c|c|}
\hline \multirow{2}{*}{ NAME } & \multirow{2}{*}{ SEX } & \multirow{2}{*}{ AGE (years) } & \multicolumn{3}{|c|}{ EXPERIMENT 1} & \multirow{2}{*}{ EXPERIMENT 2} \\
\hline & & & Control A & Control B & Test trials & \\
\hline Ettore & M & 7 & $6 / 16, \chi^{2}=1.00, p=0.32$ & $11 / 16, \chi^{2}=2.25, p=0.13$ & $5 / 16, \chi^{2}=2.25, p=0.13$ & $11 / 16, \chi^{2}=2.25, p=0.13$ \\
\hline Fulvia & $\mathrm{F}$ & 8 & $8 / 16, \chi^{2}=0.00, p=1.00$ & $10 / 16, \chi^{2}=1.00, p=0.32$ & $3 / 16, \chi^{2}=6.25, p=0.01 *$ & $13 / 16, \chi^{2}=6.25, p=0.01 *$ \\
\hline Grace & $\mathrm{F}$ & 10 & $10 / 16, \chi^{2}=1.00, p=0.32$ & $6 / 16, \chi^{2}=1.00, p=0.32$ & $6 / 16, \chi^{2}=1.00, p=0.32$ & N/A \\
\hline Jhonny & M & 7 & $8 / 16, \chi^{2}=0.00, p=1.00$ & $7 / 16, \chi^{2}=0.25, p=0.62$ & $10 / 16, \chi^{2}=1.00, p=0.32$ & $11 / 16, \chi^{2}=2.25, p=0.13$ \\
\hline Kalia & $\mathrm{F}$ & 2 & $10 / 16, \chi^{2}=1.00, p=0.32$ & $10 / 16, \chi^{2}=1.00, p=0.32$ & $10 / 16, \chi^{2}=1.00, p=0.32$ & $14 / 16, \chi^{2}=9.00, p=0.00 *$ \\
\hline Monique & $\mathrm{F}$ & 9 & $8 / 16, \chi^{2}=0.00, p=1.00$ & $8 / 16, \chi^{2}=0.00, p=1.00$ & $8 / 16, \chi^{2}=0.00, p=1.00$ & $7 / 16, \chi^{2}=0.25, p=0.62$ \\
\hline Sally & $\mathrm{F}$ & 10 & $10 / 16, \chi^{2}=1.00, p=0.32$ & $8 / 16, \chi^{2}=0.00, p=1.00$ & $10 / 16, \chi^{2}=1.00, p=0.32$ & $12 / 16, \chi^{2}=4.00, p=0.05^{*}$ \\
\hline Teide & M & 7 & $10 / 16, \chi^{2}=1.00, p=0.32$ & $9 / 16, \chi^{2}=0.25, p=0.62$ & $6 / 16, \chi^{2}=1.00, p=0.32$ & $15 / 16, \chi^{2}=12.25, p<0.00^{*}$ \\
\hline Zeno & M & 7 & $9 / 16, \chi^{2}=0.25, p=0.62$ & $7 / 16, \chi^{2}=0.25, p=0.62$ & $6 / 16, \chi^{2}=1.00, p=0.32$ & $8 / 16, \chi^{2}=0.00, p=1.00$ \\
\hline
\end{tabular}

Note: Control trials $=$ frequency of choices for the larger quantity; Test trials = frequency of choices for the portion of food on the smaller plate

*denotes a significant departure from chance at chi-square test $(p<0.05)$. 
Two different portion sizes were presented to the lemurs: The larger portion was $3 \mathrm{~cm}$ in diameter and $0.5 \mathrm{~cm}$ in height (volume $=3.53 \mathrm{~cm}^{3}$ ), whereas the smaller portion was $2.44 \mathrm{~cm}$ in diameter and 0.5 $\mathrm{cm}$ in height $\left(\right.$ volume $\left.=2.34 \mathrm{~cm}^{3}\right)$.

The subjects saw the stimuli through a transparent Plexiglas window $(32 \mathrm{~cm}$ width $\mathrm{x} 27 \mathrm{~cm}$ height) in the "choice area" (50 width x $54.5 \mathrm{~cm}$ length) and could reach the tray and select one food portion through a hole (32 width $\times 4 \mathrm{~cm}$ height). The "choice area" was connected to the entry guillotine door by a catwalk ( $75 \mathrm{~cm}$ length $\mathrm{x} 43.5$ width) of wire mesh; the entry guillotine was $108 \mathrm{~cm}$ from the ground. The catwalk was inclined by a 27 degree angle. The exit guillotine door was accessible from the ground. Figure 2 shows a schematic representation of the experimental context.

Procedure. Before starting the experiment, a short familiarization phase was set up. At this stage, a plate containing a single food portion was presented in the center of the tray. Subjects were familiarized with the opportunity to eat the contents of the plate in the choice area. This phase lasted four days, four trials each day, for a total of 16 trials.

After the familiarization phase, the experiment began. Lemurs received 12 sessions for 12 consecutive days; each session consisted of four trials, for a total of 48 trials. Sessions were comprised of both control trials and test trials (Figure 3). As in previous studies of chimpanzees (Parrish \& Beran, 2014) and dogs (Miletto Petrazzini, Bisazza, et al., 2017), two different control trials were presented: "control A" and "control B." In control A, two different food portions were presented on the two small plates, whereas in control B the two portions were presented on the two large plates (inter-plate distance was $10 \mathrm{~cm}$ ). In both control types, the ratio between the food portions was equal to 0.66. Similar ratios were commonly used in spontaneous quantity discrimination of primate and non-primate species (Banszegi, Urrutia, Szenczi, \& Hudson, 2016; Luxon-Xiccato, Miletto Petrazzini, Agrillo, \& Bisazza, 2015; Miletto Petrazzini \& Wynne, 2016; Miletto Petrazzini, Fraccaroli, et al., 2017). Control trials were set up to assess if the lemurs selected the larger portion of food in our experimental context. In test trials, equal food portions (diameter $3 \mathrm{~cm}$ ) were presented on different-sized plates, one large plate and one small plate. The ratio between the area occupied by the food portion and the smaller plate was equal to 0.67 , as it was shown that it is an optimal ratio, at least in human participants, to generate the misperception of the size commonly reported in the Delboeuf illusion (Piaget, 1957). The order of presentation in the control and test trials was varied according to a pseudo-random sequence as done in other preference tests (e.g., Miletto Petrazzini, Bisazza, et al., 2017). However, each daily session always started with a control trial. The position (left/right) of different-sized portions was counterbalanced over the trials to inhibit side biases. In particular, the larger food portion in the control trials was never presented more than twice in a row on the same side. Also, a single test trial was always preceded and followed by a control trial. The order of presentation in the trials was identical for all subjects.

Two experimenters were involved in the experiment. One experimenter prepared the plates in the trays, and the other presented the trays to the subjects and controlled their entrance into the test room and their exit. At the beginning of each session, one experimenter enclosed the lemurs in the indoor area by attracting them with apple slices, while the other experimenter arranged the food portions on both plates in all of the trays out of the lemurs' sight. The entry order of the lemurs in the test room changed each session according to their motivation. Once the subject entered the test room, it was attracted to the center of the choice area through a small piece of primate jelly. After the subject had sat, the experimenter presented the first tray. The lemurs could see the plates for $5 \mathrm{~s}$ before making their choice (defined as the first food portion touched by the animal). However, if the subjects' hands were not on the platform of the choice area, the experimenter waited to make the contents of the tray available until both hands were on the platform. This procedure limited the possibility that subjects simply selected the array closest to their hands. Once the subject had chosen one food portion, it was allowed to grab and eat it while the tray with the other stimulus was quickly removed by the experimenter (see Supplemental Material for an example of a trial). 


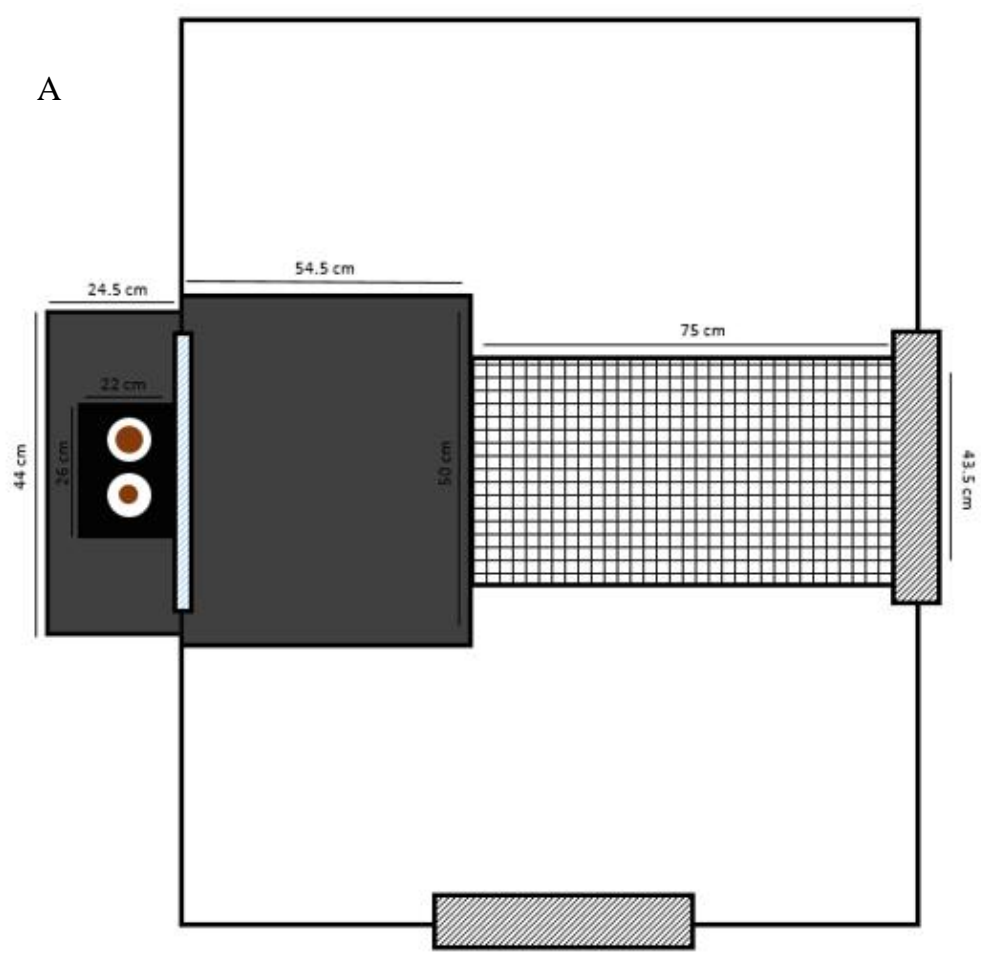

\section{B}
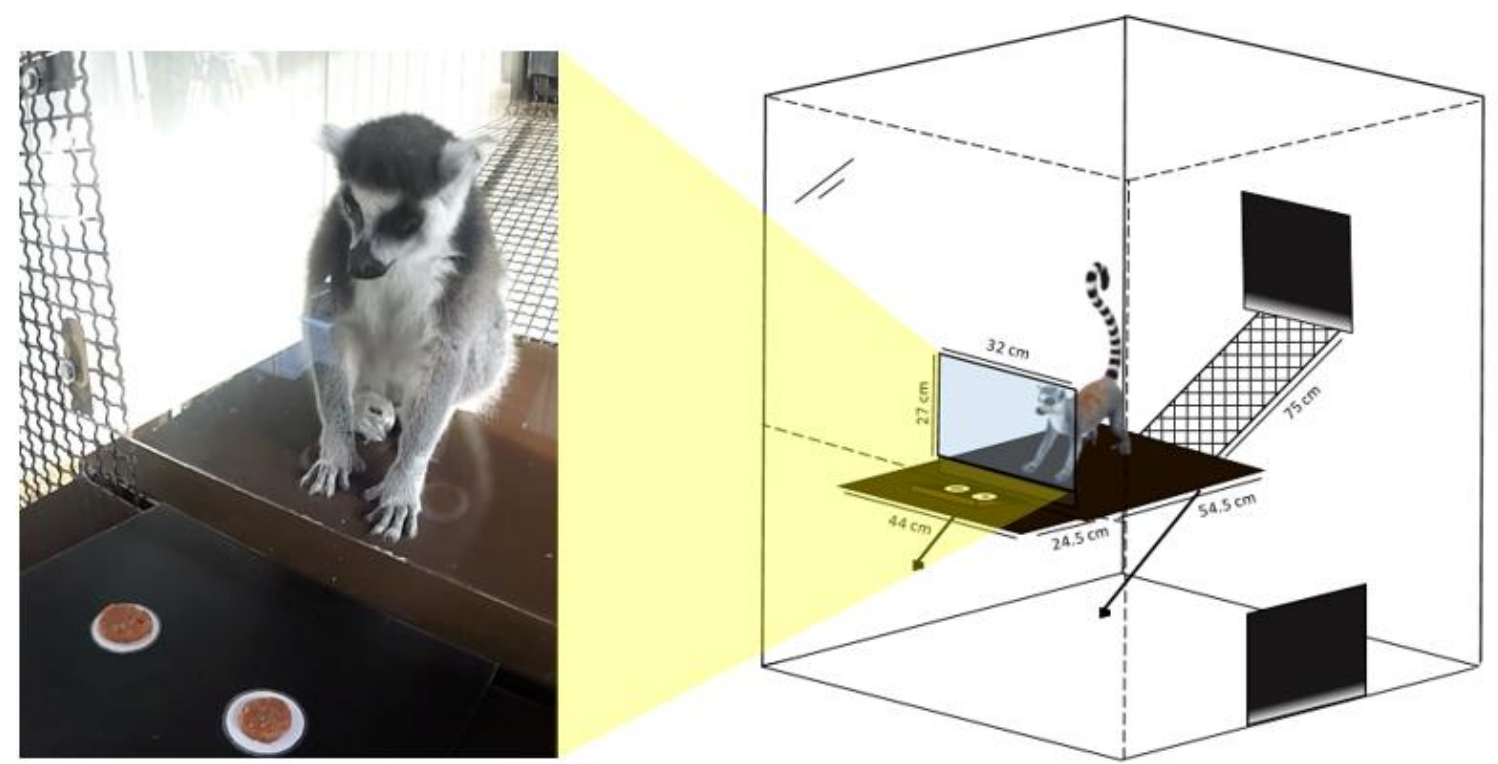

Figure 2. Experimental setup. Subjects entered the choice area by walking on a wire mesh. Stimuli were presented in a tray visible through a transparent Plexiglas window. As the dependent variable we recorded the first food portion touched by the animal in each trial. Aerial (A) and lateral (B) view of the experimental context is visible here. The picture shows Kalia engaged in a control trial of Experiment 1. 
Santacà et al. 371

A

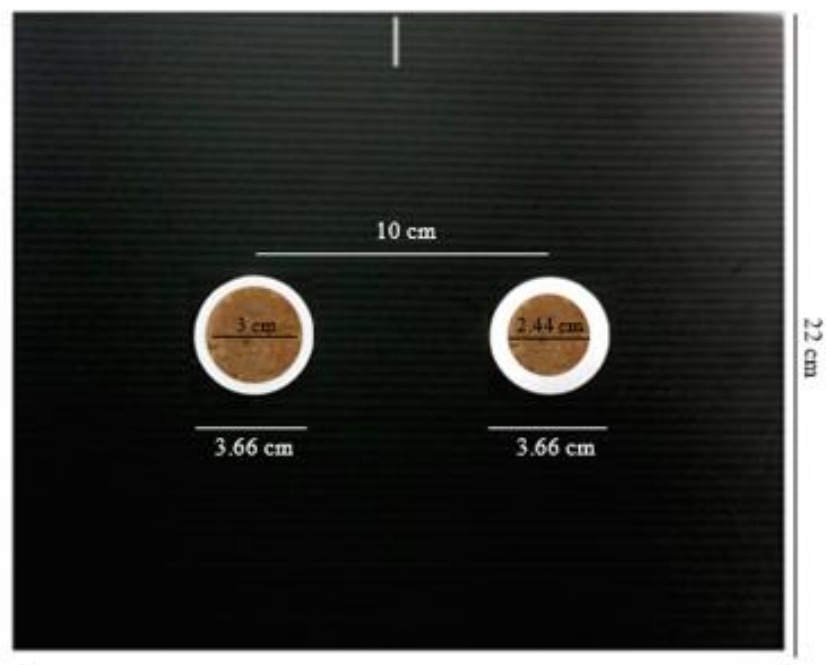

B

B

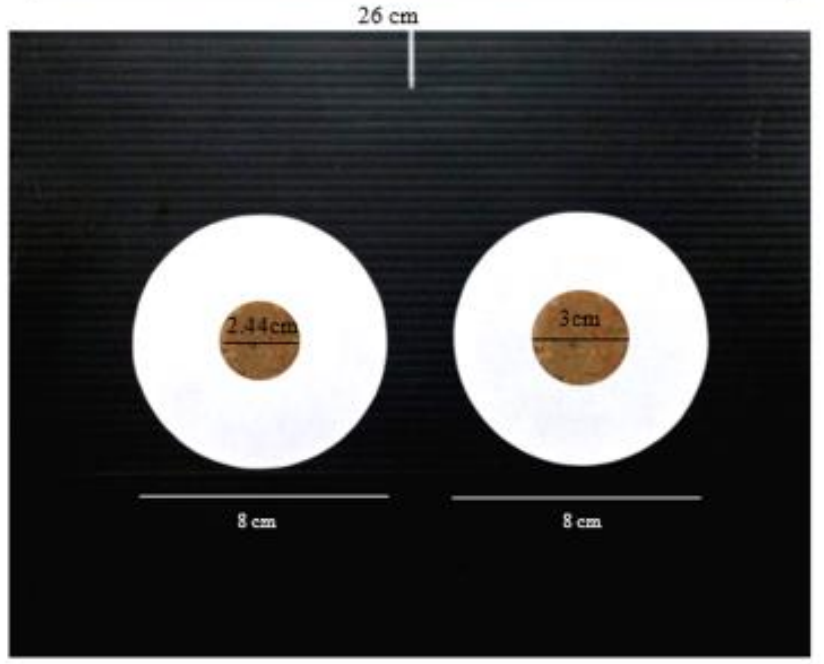

C

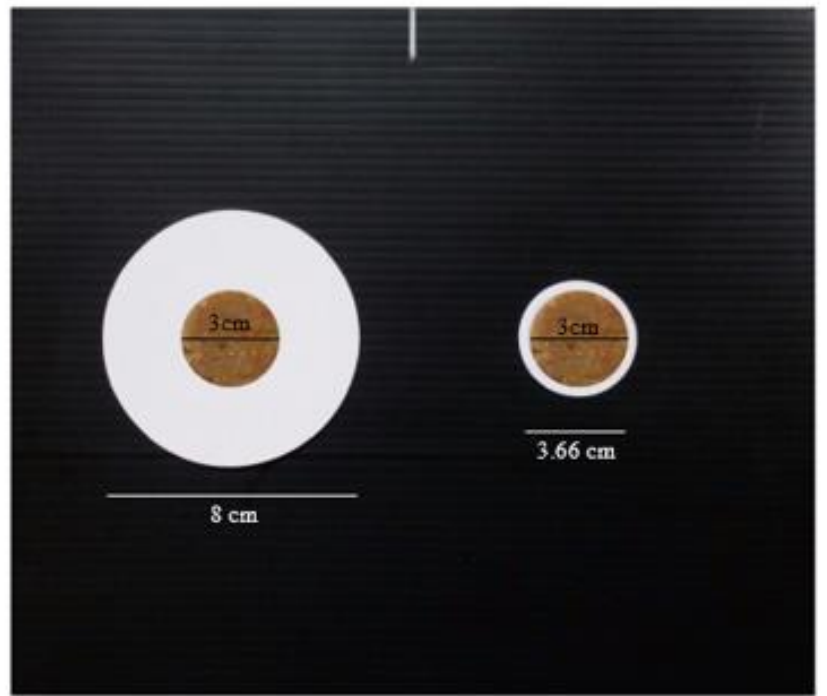

Figure 3. Stimuli of Experiment 1. Two plates containing different or equal-sized food portions were presented: (A) control trial A with different food portions in two identical small plates; (B) control trial B with different food portions in two identical large plates; $(\mathbf{C})$ test trials with equal-sized food portions in two plates differing in size. 
After the daily session was completed, the exit guillotine was opened and the subject could exit to the outdoor area where it received a peanut. Trials were conducted depending upon the lemurs' motivation. Choices were mostly unambiguous; the experimenter noted the subject's response after each trial and then set up a new trial. However, each session was also recorded, and the videos were subsequently analyzed to note the subjects' choices in case of ambiguous responses. To assess interobserver reliability, another observer, blind to the experimental condition, analyzed the videos and scored the lemurs' choices (20\% of overall trials). An excellent interobserver agreement was found (Pearson's correlation $r=1.0, p<0.0001$ ).

Statistical analyses were conducted using SPSS 24.0. Individual analyses were performed on the frequency of choices for the larger quantity of food (control trials) or for the portion of food on the smaller plate (test trials) by using chi-square tests. In the absence of previous studies on size illusions in prosimians, we could not make any a-priori predictions, hence all statistical tests were two-tailed; $\alpha$ was set at 0.05 .

Even though we are aware that the small sample size in group analyses does not permit us to draw firm conclusions, we also analyzed the overall performance of subjects. As data were normally distributed (Shapiro-Wilk test, $p>0.05$ ), they were analyzed using parametric statistics. The proportion of choices for the larger quantity of food was used in control trials; in test trials we analyzed the proportion of choices for the portion of food on the smaller plate. To assess if the lemurs were able to discriminate between the two quantities in control trials or if the lemurs selected one plate more than chance in test trials, we performed one-sample t-tests (chance level was 0.5). A paired t-test was used to compare performance in the two control trials.

\section{Results}

Control trials. Individual analyses (chi-square tests) on the frequency of choices for the larger food portion showed that no subjects selected one plate more than chance in either control A or control B (Table 1). The lack of significant preference for any array was further demonstrated by group analyses: No preference was found in either control A (mean: 0.549, 95\% CI [0.482, 0.616]; one-sample t-test $t(8)$ $=1.673, p=0.133$ ) or control B (mean: $0.528,95 \%$ CI $[0.448,0.608], t(8)=0.800, p=0.447$ ). No difference was found between the two conditions (paired t-test $t(8)=0.385, p=0.710$ ).

Test trials. No choice for either plate was found at the individual level for any subject, with the exception of one (Fulvia) who selected the larger plate containing food in 13 out of 16 trials, the opposite to what would be expected from a human-like perception of the illusion (Table 1). Group analyses confirmed that lemurs did not significantly more often choose one food plate over the other (mean: 0.444 , $95 \%$ CI $[0.323,0.566], t(8)=-1.057, p=0.321$, Figure 4$)$.

\section{Experiment 2}

Experiment 1 does not provide any evidence supporting the perception of the Delboeuf illusion in lemurs. Above all, the lack of significant choice for the larger food portion in control trials does not permit us to draw any conclusion about lemurs' sensitivity to this illusion. Indeed, even though we adapted the experimental procedure successfully used in apes (Parrish \& Beran, 2014), the possibility exists that the present experimental setting was not optimal for testing spontaneous quantity discrimination in this species. To test this hypothesis, in Experiment 2 we enlarged the physical difference between food portion, 0.47 instead of 0.66. This experiment, unfortunately, does not provide a better understanding of lemurs' sensitivity to the Delboeuf illusion. However, if lemurs exhibit a significant discrimination of the larger food portion, this would imply that lemurs have a spontaneous tendency to maximize food intake. Hence, the present experimental setting would be at least adequate for testing spontaneous size discrimination in this species. This might represent a starting point for future investigations on the size discrimination threshold. 


\section{Method}

Subjects, stimuli, apparatus and procedure. We tested the same subjects involved in Experiment 1 with the exception of Grace who ceased to participate after Experiment 1. Stimuli, apparatus and procedures were identical with four exceptions. No test trials with the illusory pattern were presented. All trials presented a 0.47 ratio between the smaller and the larger food portion (diameter larger portion: $3 \mathrm{~cm}$; diameter smaller portion: $2.06 \mathrm{~cm}$; height of both portions: $0.5 \mathrm{~cm}$; volume of the larger portion: $3.53 \mathrm{~cm}^{3}$; volume of the smaller portion: $1.67 \mathrm{~cm}^{3}$ ). In half of the trials food was presented on two small plates (a condition previously called "control A" in Experiment 1), and in the other half of the trials food was presented on two large plates (a condition previously called "control B"). However, as Experiment 1 showed no difference in subjects' choices as a function of the type of control trial, we presented 16 overall trials (eight for the first set, eight for the second one, instead of 16 trials for each set). Lastly, in order to ensure that lemurs were highly motivated to optimize food intake in each trial, each lemur performed only one trial per day (instead of four trials in each day, as done in Experiment 1).

Data were normally distributed (Shapiro-Wilk test, $p>0.05$ ), hence we used parametric statistics for group analyses.

\section{Results}

We found no difference between control $\mathrm{A}$ and control B (Control A, mean: $0.688,95 \% \mathrm{CI}$ [0.562, 0.812]; Control B, mean: 0.734, 95\% CI [0.554, 0.915]; paired t-test $t(7)=-1.000, p=0.351)$. As a consequence, for individual and group analyses, we pooled data together. Individual analyses on the frequency of choices for the larger food portion showed that four out of eight subjects selected the larger food portion significantly more often (Table 1). We found no difference in the presence of the illusory pattern (test trials of Experiment 1) between subjects able to discriminate 0.47 ratio (mean: $0.453,95 \%$ CI $[0.115,0.792]$ ) and those that were not able to discriminate it (mean: $0.453,95 \%$ CI [0.233, 0.674], independent t-test, $t(6)=0, p=1.0)$. Group analyses showed a significant discrimination of the larger quantity (mean: $0.711,95 \%$ CI $[0.565,0.856] ; t(7)=3.441, p=0.011$, Figure 4 ).

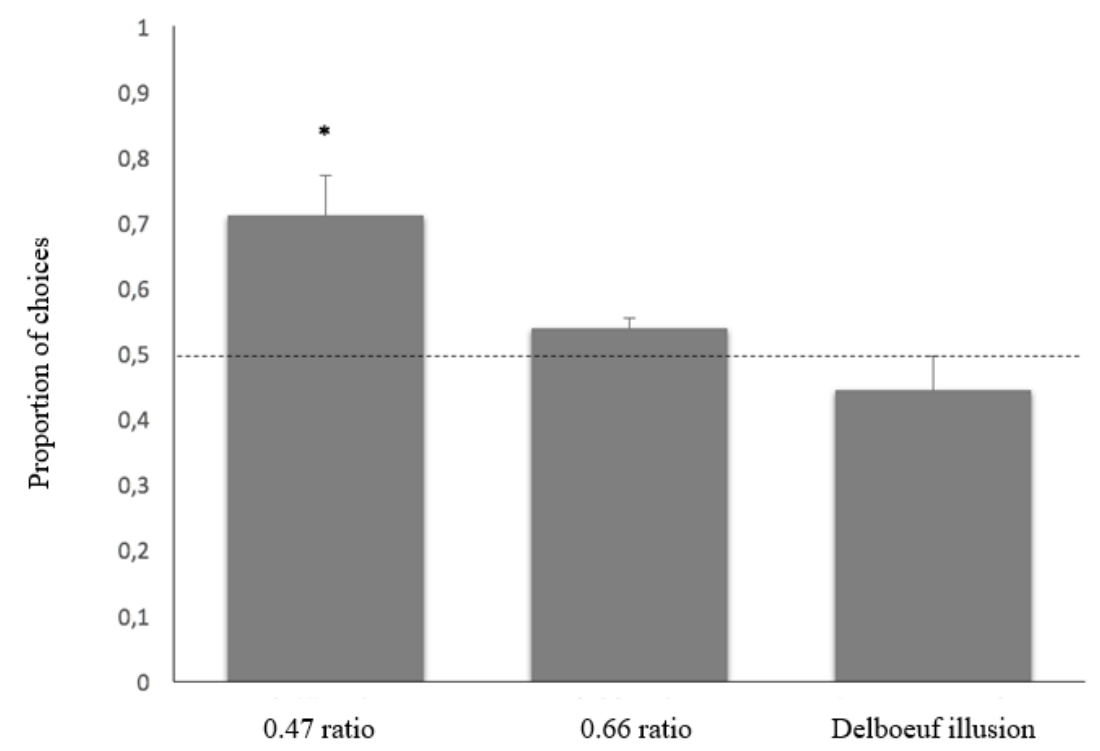

Figure 4. Results of Experiment 1 and Experiment 2. The Y-axis refers to the proportion of choices for the larger food portion in the control trials of Experiment 1 (ratio 0.66) and trials of Experiment 2 (0.47), and the proportion of choices for the food portion included in the smaller plate in the test trials with the illusory pattern (Experiment 1). Lemurs selected the larger food portion in a 0.47 but not a 0.66 ratio. No choice for either plate was shown in the presence of the illusory pattern. Bars represent the standard error. The asterisk $(*)$ denotes a significant departure from chance level. 


\section{Discussion}

The present study represents the first attempt to investigate whether prosimians perceive visual illusions. To achieve this goal, we adapted the procedure recently used with chimpanzees (Parrish \& Beran, 2014) and dogs (Miletto Petrazzini, Bisazza, et al., 2017) for the study of one of the most commonly perceived size illusions in human literature, the Delboeuf illusion.

In Experiment 1, individual and group analyses of control trials indicated no significant choice for either array. The lack of discrimination of the larger array suggests that a 0.66 ratio may not be detected by lemurs, although of course further investigation is needed. These results, however, do not permit us to draw any firm conclusion about the performance exhibited in test trials. In line with control trials, individual and group analyses of test trials showed that lemurs did not select one plate significantly more often when presented with the illusory pattern. A lack of choice for the stimulus encircled by the smaller ring has been previously found in dogs, and it is commonly assumed as evidence of a lack of sensitivity for this size illusion (Byosere et al., 2017; Miletto Petrazzini, Bisazza, et al., 2017). The only exception in our study was represented by Fulvia, who consistently selected the larger plate. This might lead us to believe that she perceived a reverse illusion, as previously reported in other non-human species (Nakamura et al., 2008; Watanabe et al., 2011). However, we do not feel this is the most likely explanation of her performance, as Fulvia did not show any significant discrimination in either control A or control B. Our best explanation is that Fulvia was highly sensitive to trials in which the plates were differing in size. This condition was less frequent compared to control trials with identical plates, and it may have led to a bias for choosing one array (in this case, the food portion on the larger plate), which was not observed when identical plates were presented.

The main conclusion from Experiment 1 is that our experimental setting was not sensitive enough to investigate lemurs' ability to discriminate between two items differing in size. Alternatively, the null results could be due to lack of lemurs' precision in size judgments in the presence of a 0.66 ratio. It is worth noting that we adapted the procedure successfully used in the past for the study of the Delboeuf illusion in mammals (Miletto Petrazzini, Bisazza, et al., 2017; Parrish \& Beran, 2014). The null results here obtained cannot permit us to draw conclusions about the perception of the Delboeuf illusion in this species, but they are still informative because they clearly show that this methodological approach is not adequate to investigate the Delboeuf illusion in lemurs. The critical role of the experimental context was recently highlighted by Parrish et al. (2015). Monkeys' sensitivity to the Delbouef illusion varied as a function of two methodological variables: the ratio of the target stimulus diameter to concentric ring diameter and the type of task (absolute vs. relative size discrimination). Researchers have recently remarked on the importance of reducing methodological variability to assess similarities and differences in perceptual and cognitive abilities of vertebrates (for reviews see Agrillo \& Bisazza, 2014; Feng, Chouinard, \& Howell, 2016). However, we must point out that even when animals are tested in identical conditions, other concurrent factors intrinsically related to the tested species may prevent a reliable comparison. For instance, lemurs' visual acuity seems to be lower than that of apes (Feng et al., 2016; Neuringer, Kosobud, \& Cochrane, 1981). As a consequence, comparing the performance of apes and lemurs with visual stimuli presented in similar environmental conditions (e.g., similar distance and visual angles) may be inappropriate. Multiple experimental approaches testing the same hypothesis with different stimuli and environmental conditions are needed to better compare the visual perception of various mammals.

The lack of choice in Experiment 1 may bring us to believe that the present procedure is totally inadequate to study lemurs' quantitative abilities. Previous studies showed that untrained lemurs can spontaneously select the larger quantity of food (e.g., Reddy, MacLean, Sandel, \& Hare, 2015; Santos, Barnes, \& Mahajan, 2005; Stevens \& Mühlhoff, 2012). In these studies, subjects were presented with multiple items (e.g., 2 vs. 4). In a study by Lewis, Jaffe and Brannon (2005) lemurs proved able to discriminate up to a 0.50 ratio; a lower performance was described by Jones and Brannon (2012), with lemurs discriminating a 0.33 but not a 0.50 ratio. Different authors found that the precision in quantitative judgments is different when subjects are required to discriminate between multiple items (e.g., 2 vs. 4 
with 0.50 ratio) compared to when numerical information is made irrelevant and only total area can be used as a discriminative cue (e.g., one large vs. one small piece of food, ratio 0.50; Cordes \& Brannon, 2011; Lucon-Xiccato et al., 2015; Miletto Petrazzini, Fraccaroli, et al., 2017; Piffer, Miletto Petrazzini, \& Agrillo, 2013). This is presumed to reflect the involvement of different cognitive abilities in the two types of tasks: numerical abilities in the presence of multiple items and spatial abilities in the presence of two items differing in size.

In Experiment 2 we decided to enlarge the physical difference between the food portions (0.47) to establish whether lemurs try to optimize food intake in a task not involving numerical systems. Lemurs showed an overall ability to discriminate this ratio, although only $50 \%$ of the subjects discriminated the larger food portion at an individual level. The inter-individual variability here observed suggests that this ratio is not easily discriminated by this species.

Further research is needed. Provided that lemurs can discriminate at least a 0.47 ratio, it would be important to determine the threshold for the spontaneous discrimination of the larger food portion. Larger physical differences between the two plates in the illusory array might also be presented to see whether subjects perceive any kind of size illusion. Apart from spontaneous choice tests, the use of training procedures would be fundamental to see if lemurs can be trained to detect subtle size differences as those required in the presence of the Delboeuf illusion. For instance, in the study by Byosiere et al. (2017) dogs underwent extensive training requiring them to make fine size discriminations before starting test trials with the illusory pattern. The lack of choice shown by trained dogs in the test phase integrated the conclusion of the spontaneous choice tests made in the same species by Miletto Petrazzini, Bisazza, et al. (2017).

At present we cannot perform these studies. One of the prerequisites for spontaneous choice tests is that subjects be tested in a limited number of trials in order to observe the natural response of untrained animals and prevent any form of learning across trials (see Agrillo \& Bisazza, 2014). In Experiment 1 and Experiment 2 we tested all the members of the colony of ring-tailed lemurs housed in the park, hence no naïve subjects were available. In addition, unlike other primates, training studies with lemurs have only recently appeared in the literature (e.g., MacLean, Merritt \& Brannon, 2008; Merritt, MacLean, Crawford $\&$ Brannon, 2011), and we are not currently equipped for this type of investigation.

Unfortunately, until those data are available, we cannot draw any conclusion about the perceptual biases affecting the precision of lemurs' relative size judgments. However, our investigation helped us to advance our understanding of lemurs' size judgments. We found that lemurs attempt to maximize their food intake in the presence of two items differing in size, and they can solve this task when approximately a two-fold difference is presented. In addition, we showed that the procedure successfully used by Parrish and Beran (2014) cannot be applied to ring-tailed lemurs given the low precision exhibited by subjects in control trials. Hopefully, this will help researchers to determine the most appropriate procedure to further investigate lemurs' size illusions.

\section{Acknowledgements}

This study complies with all laws of the country (Italy) in which it was performed. It was supported by a "FIRB grant 2013" (prot.: RBFR13KHFS) awarded to C. Agrillo and by a "PRIN grant 2015" (prot.: 2015FFATB7) awarded to A. Bisazza from Ministero dell'Istruzione, Università e Ricerca (MIUR, Italy). We would like to thank Dr. Cesare Avesani Zaborra and Dr. Camillo Sandri, Parco Natura Viva CEO and general curator respectively, for allowing this study to take place.

\section{References}

Agrillo, C., \& Bisazza, A. (2014). Spontaneous versus trained numerical abilities. A comparison between the two main tools to study numerical competence in non-human animals. Journal of Neuroscience Methods, 234, $82-91$.

Agrillo, C., Parrish, A. E., \& Beran, M. J. (2014a). Do rhesus monkeys (Macaca mulatta) perceive the Zöllner 
illusion? Psychonomic Bulletin \& Review, 21, 986 - 994.

Agrillo, C., Parrish, A. E., \& Beran, M. J. (2014b). Do primates see the solitaire illusion differently? A comparative assessment of humans (Homo sapiens), chimpanzees (Pan troglodytes), rhesus monkeys (Macaca mulatta), and capuchin monkeys (Cebus apella). Journal of Comparative Psychology, 128, $402-413$.

Banszegi, O., Urrutia, A., Szenczi, P., \& Hudson, R. (2016). More or less: Spontaneous quantity discrimination in the domestic cat. Animal Cognition, 19, $879-888$.

Bayne, K. L., \& Davis, R. (1983). Susceptibility of rhesus monkeys (Macaca mulatta) to the Ponzo illusion. Bulletin of the Psychonomic Society, 21, 476 - 478.

Byosiere, S. E., Feng, L. C., Woodhead, J. K., Rutter, N. J., Chouinard, P. A., Howell, T. J., \& Bennett, P. C. (2017). Visual perception in domestic dogs: Susceptibility to the Ebbinghaus-Titchener and Delboeuf illusions. Animal Cognition, 20, 435 - 448.

Cordes, S., \& Brannon, E. M. (2011). Attending to one of many: When infants are surprisingly poor at discriminating an item's size. Frontiers in Psychology, 2, 65.

Davis, B., Payne, C. R., \& Bui, M. (2016). Making small food units seem regular: How larger table size reduces calories to be consumed. Journal of the Association of Consumer Research, 1, $115-124$.

Feng L. C., Chouinard P. A., Howell T. J., \& Bennett P. C. (2017). Why do animals differ in their susceptibility to geometrical illusions? Psychonomic Bulletin \& Review, 24, 262 - 276.

Fujita, K. (1997). Perception of the Ponzo illusion by rhesus monkeys, chimpanzees, and humans: Similarity and difference in the three primate species. Perception \& Psychophysics, 59, $284-292$.

Fuss, T., Bleckmann, H., \& Schluessel, V. (2014). The brain creates illusions not just for us: Sharks (Chiloscyllium griseum) can "see the magic" as well. Frontiers in Neural Circuits, 8, 24.

Jones, S. M., \& Brannon, E. M. (2012). Prosimian primates show ratio dependence in spontaneous quantity discriminations. Frontiers in Psychology, 3, 550. doi: 10.3389/fpsyg.2012.00550

Lewis, K., Jaffe, S., \& Brannon, E. M. (2005). Analog number representations in mongoose lemurs (Eulemur mongoz): Evidence from a search task. Animal Cognition, 8, 247-252.

Lucon-Xiccato, T., Miletto Petrazzini, M. E., Agrillo, C., \& Bisazza, A. (2015). Guppies discriminate between two quantities of food items but prioritize item size over total amount. Animal Behaviour, 107, 183 - 191.

MacLean, E. L., Merritt, D. J., \& Brannon, E. M. (2008). Social complexity predicts transitive reasoning in prosimian primates. Animal Behaviour, 76, $479-486$.

Merritt, D. J., Maclean, E. L., Crawford, J. C., \& Brannon, E. M. (2011). Numerical rule-learning in ring-tailed Lemurs (Lemur catta). Frontiers in Psychology, 2, 23. doi: 10.3389/fpsyg.2011.00023

Miletto Petrazzini, M. E., Bisazza, A., \& Agrillo, C. (2017). Do domestic dogs (Canis lupus familiaris) perceive the Delboeuf illusion? Animal Cognition, 20, 427 - 434.

Miletto Petrazzini, M. E., Fraccaroli, I., Gariboldi, F., Agrillo, C., Bisazza, A., Bertolucci, C., \& Foà, A. (2017). Quantitative abilities in a reptile (Podarcis sicula). Biology Letters, 13, 20160899.

Miletto Petrazzini, M. E., \& Wynne, C. D. (2016). What counts for dogs (Canis lupus familiaris) in a quantity discrimination task? Behavioural Processes, 122, 90 - 97.

Nakamura, N., Watanabe, S., \& Fujita, K. (2008). Pigeons perceive the Ebbinghaus-Titchener circles as an assimilation illusion. Journal of Experimental Psychology: Animal Behavior Processes, 34, 375 - 387.

Neuringer, M., Kosobud, A., \& Cochrane, G. (1981). Visual acuity of Lemur catta, a diurnal prosimian. Investigative Ophthalmology and Visual Science, 20, 49.

Parrish, A. E., Agrillo, C., Perdue, B. M., \& Beran, M. J. (2016). The elusive illusion: Do children (Homo sapiens) and capuchin monkeys (Cebus apella) see the Solitaire illusion? Journal of Experimental Child Psychology, 142, $83-95$.

Parrish, A. E., \& Beran, M. J. (2014). When less is more: Like humans, chimpanzees (Pan troglodytes) misperceive food amounts based on plate size. Animal Cognition, 17, $427-434$.

Parrish, A. E., Brosnan, S. F., \& Beran, M. J. (2015). Do you see what I see? A comparative investigation of the Delboeuf illusion in humans (Homo sapiens), rhesus monkeys (Macaca mulatta), and capuchin monkeys (Cebus apella). Journal of Experimental Psychology: Animal Learning and Cognition, 41, 395 - 405.

Pepperberg, I. M., Vicinay, J., \& Cavanagh, P. (2008). Processing of the Müller-Lyer illusion by a grey parrot (Psittacus erithacus). Perception, 37, $765-781$.

Piaget, J. (1957). Les mécanismes perceptifs. Paris: Presses Universitaires de France.

Piffer, L., Miletto Petrazzini, M. E., \& Agrillo, C. (2013). Large number discrimination in newborn fish. PLoS ONE, 8, e62466.

Reddy, R. B., MacLean, E. L., Sandel, A. A., \& Hare, B. (2015). Social inhibitory control in five lemur species. Primates, 56, 241 - 252. 
Reuter, K. E., LaFleur, M., \& Clarke, T. A. (2017). Endangered species: Illegal lemur trade grows in Madagascar. Nature, 541, 157.

Rosa Salva, O., Rugani, R., Cavazzana, A., Regolin, L., \& Vallortigara, G. (2013). Perception of the Ebbinghaus illusion in four-day-old domestic chicks (Gallus gallus). Animal Cognition, 16, 895 - 906.

Santos, L. R., Barnes, J. L., \& Mahajan, N. (2005). Expectations about numerical events in four lemur species (Eulemur fulvus, Eulemur mongoz, Lemur catta and Varecia rubra). Animal Cognition, 8, 253 - 262.

Stevens, J. R., \& Mühlhoff, N. (2012). Intertemporal choice in lemurs. Behavioural Processes, 89, 121 - 127.

Suganuma, E., Pessoa, V. F., Monge-Fuentes, V., Castro, B. M., \& Tavares, M. C. H. (2007). Perception of the Müller-Lyer illusion in capuchin monkeys (Cebus apella). Behavioural Brain Research, 182, 67 - 72.

Van Ittersum, K., \& Wansink, B. (2007). Do children really prefer large portions? Visual illusions bias their estimates and intake. Journal of the American Dietetic Association, 107, 1107 - 1110.

Wansink, B., \& Cheney, M. M. (2005). Super bowls: Serving bowl size and food consumption. The Journal of the American Medical Association, 293, 1727 - 1728.

Watanabe, S., Nakamura, N., \& Fujita, K. (2011). Pigeons perceive a reversed Zöllner illusion. Cognition, 119, 137 -141 .

Watanabe, S., Nakamura, N., \& Fujita, K. (2013). Bantams (Gallus gallus domesticus) also perceive a reversed Zöllner illusion. Animal Cognition, 16, 109 - 115. 